Barnes-Scheufler et al. Attentional Competition during VWM Encoding in Schizophrenia

\title{
Impaired and Intact Aspects of Attentional Competition and Prioritization during Visual Working Memory Encoding in Schizophrenia
}

Running Title: Attentional Competition during VWM Encoding in Schizophrenia

Catherine V. Barnes-Scheufler MSc ${ }^{1}$, Lara Rösler PhD ${ }^{1,2}$, Michael Schaum MSc ${ }^{3}$, Carmen Schiweck $\mathrm{PhD}^{1}$, Benjamin Peters $\mathrm{PhD}^{4,5}$, Jutta S. Mayer $\mathrm{PhD}^{6}$, Andreas Reif $\mathrm{MD}^{1}$, Michael Wibral $\mathrm{PhD}^{7}$, Robert A. Bittner MD ${ }^{1,8 *}$

${ }^{1}$ Department of Psychiatry, Psychosomatic Medicine, and Psychotherapy, University Hospital Frankfurt, Germany

${ }^{2}$ Netherlands Institute for Neuroscience, Amsterdam, The Netherlands

${ }^{3}$ Leibniz Institute for Resilience Research, Mainz, Germany

${ }^{4}$ Institute of Medical Psychology, Goethe University Frankfurt, Germany

${ }^{5}$ Zuckerman Mind Brain Behavior Institute, Columbia University, New York, NY, USA

${ }^{6}$ Department of Child and Adolescent Psychiatry, Psychosomatics and Psychotherapy, University Hospital Frankfurt, Germany

${ }^{7}$ Campus Institute for Dynamics of Biological Networks, Georg-August University, Göttingen, Germany

${ }^{8}$ Ernst Strüngmann Institute (ESI) for Neuroscience in Cooperation with Max Planck Society, Frankfurt, Germany

${ }^{*}$ Corresponding author. Department of Psychiatry, Psychosomatic Medicine and Psychotherapy, University Hospital Frankfurt, Goethe University, HeinrichHoffmann-Str. 10, D-60528 Frankfurt am Main, Germany. 
Barnes-Scheufler et al. Attentional Competition during VWM Encoding in Schizophrenia

Phone: +49696301 84713. Fax: +4969630181775.

Email: robert.bittner@kgu.de

\section{Word Count}

Total number of words in text: 5114

Total number of words in main body: 3507

Total number of words in abstract: 220

Number of Figures: 2

Number of Tables: 2

Disclosure: We report no financial relationships with commercial interests. C.V Barnes-Scheufler was supported by a "main doctus" scholarship from The Polytechnic Foundation of Frankfurt am Main. The Authors have declared that there are no conflicts of interest in relation to the subject of this study.

\section{ORCID iDs}

C.V.B.-S.: orcid.org/0000-0002-4730-1981

L.R.: orcid.org/0000-0003-1046-1073

M.S.: orcid.org/0000-0002-6589-4530

C.S.: orcid.org/0000-0003-4215-0448

B.P.: orcid.org/0000-0002-0948-8976

J.S.M.: orcid.org/0000-0002-5328-5893

A.R.: orcid.org/0000-0002-0992-634X

M.W.: orcid.org/0000-0001-8010-5862

R.A.B.: orcid.org/0000-0003-2021-0358 
Barnes-Scheufler et al. Attentional Competition during VWM Encoding in Schizophrenia

\section{ABSTRACT}

\section{Objective}

People with schizophrenia (PSZ) are impaired in the attentional prioritization of nonsalient but relevant stimuli over salient but irrelevant distractors during visual working memory (VWM) encoding. Conversely, the guidance of top-down attention by external predictive cues is intact. Yet, it is unknown whether this preserved ability can help PSZ overcome impaired attentional prioritization in the presence of salient distractors.

\section{Methods}

We employed a visuospatial change-detection task using four Gabor Patches with differing orientations in 69 PSZ and 74 healthy controls (HCS). Two patches flickered to reflect saliency and either a predictive or a non-predictive cue was displayed resulting in four conditions.

\section{Results}

Across all conditions, PSZ stored significantly less information in VWM than HCS (all $p<0.001$ ). With a non-predictive cue, PSZ stored significantly more salient than non-salient information $\left(\mathrm{t}_{140}=5.66, p<0.001, d t=0.5\right)$. With a predictive cue, PSZ stored significantly more non-salient information ( $\left.t_{140}=5.70, p<0.001, d t=0.5\right)$.

\section{Conclusion}

Our findings support a bottom-up bias in schizophrenia with performance significantly better for visually salient information in the absence of a predictive cue. These results indicate that bottom-up attentional prioritization is disrupted in schizophrenia, but the top-down utilization of cues is intact. We conclude that additional top-down information significantly improves performance in PSZ when non-salient visual information needs to be encoded in working memory. 
Barnes-Scheufler et al. Attentional Competition during VWM Encoding in Schizophrenia

\section{INTRODUCTION}

Visuospatial working memory (VWM) and attention are two intricately and bidirectionally related cognitive domains (1-3) essential for elucidating the basis of perturbed information processing in schizophrenia (4). The role of top-down attention for the initial encoding of VWM representations is particularly crucial due to clear behavioral $(5-7)$ and neurophysiological $(8,9)$ evidence for a primary impairment of this WWM component process in people with schizophrenia (PSZ).

Specifically, top-down attention is essential for selecting goal-relevant information to be stored in WWM and the efficiency of this mechanism has a considerable influence on WWM capacity $(10,11)$. During VWM encoding, top-down attention, which is driven by stimulus relevance, competes with bottom-up attention, which is driven by stimulus salience $(12,13)$. If multiple items compete for limited processing in visual attention, selection of relevant and inhibition of irrelevant information depends on the successful execution of two top-down control processes (14). First, the control of selection aids the identification of relevant information, which should be selected (15). Second the implementation of selection enables the differential processing of selected and unselected information (15). Neural computations during attentional competition assign a distinct priority to each stimulus, which is the combined representation of its salience and behavioral relevance (16). To this end, the control of attentional selection is guided by an attentional set, which induces a top-down attentional bias toward relevant information based on current goals (17). According to the signal suppression hypothesis, this also facilitates the suppression of automatic attentional capture by visually salient distractors through inhibitory mechanisms $(18,19)$, a key element of the implementation of selection and a crucial prerequisite for adequate attentional prioritization. 
Barnes-Scheufler et al. Attentional Competition during VWM Encoding in Schizophrenia

Within the framework of predictive coding the attentional set - an internally generated high-level prediction of behavioral relevance - would be regarded as a prior. Predictive coding implies that during information processing a prior is combined with sensory information (likelihood) to compute a posterior probability (posterior) (20). Thus, for the computation of stimulus priority (posterior) during attentional competition, the attentional set is weighed against stimulus salience, which represents the likelihood of attentional capture. Notably, converging evidence indicates that schizophrenia is characterized by a decreased precision and stability of priors relative to sensory data (21), which might be related to attentional dysfunction.

Behavioral VWM experiments using flickering stimuli as part of an encoding array clearly indicate a strong attentional bias in PSZ toward highly salient stimuli, even if they are behaviorally irrelevant (5). Consequently, these stimuli are encoded into WM with inadequately high priority. The correlation between the magnitude of this bottom-up attentional bias and reduced VWM capacity (5) underscores its importance for VWM dysfunction. Overall, the bottom-up attentional bias surpasses the top-down bias induced by the attentional set. This results in a failure to adequately prioritize non-salient but relevant stimuli over salient but irrelevant distractors. These findings indicate a specific impairment in the implementation of selection in PSZ when top-down control is needed to overcome salient distractors. Importantly, control of attentional selection relying solely on an internally generated attentional set is also disturbed in PSZ (22). Additionally, the selection of relevant and inhibition of irrelevant information is impaired in PSZ when top-down control is required in order to overcome an internally generated attentional set rather than stimulus salience and this impairment is associated with their reduced WWM capacity (23). These findings are compatible with an impairment to adequately 
Barnes-Scheufler et al. Attentional Competition during VWM Encoding in Schizophrenia

utilize the attentional set as a high-level prior, particularly in the presence of highly salient distractors.

Conversely, patients' ability to utilize external spatial cues to guide top-down attention during VWM encoding and to prioritize information correctly without the presence of salient distractors is intact (24). These findings are indicative of an 'island of preserved cognitive function' (25), which can provide important clues about the cognitive and neurophysiological mechanisms underlying attentional and VWM encoding impairments in schizophrenia. The successful use of spatial cues indicates that additional visuospatial information can enhance the precision of patient's priors, and that their general ability to utilize priors is intact. This would also imply that insufficiently precise and stable priors are a primary cause of impaired attentional control in schizophrenia. However, to our knowledge, it has not yet been investigated, whether top-down attentional control of PSZ aided by external cues can prevail when directly challenged by highly salient distractors. Illuminating this issue is crucial for models of impaired attentional control and stimulus prioritization during VWM encoding. In addition to predictive coding accounts of attentional dysfunction, impaired inhibition due to disturbances in the GABAergic system - a central pathophysiological mechanism in schizophrenia - is also highly pertinent for this question.

There is converging evidence that the suppression of attentional capture by salient stimuli relies on a top-down inhibitory mechanism inducing local inhibition of such stimuli in early visual areas (26). While the exact mechanism underlying topdown controlled attentional inhibition remains unknown, there is clear evidence for an important role of inhibitory GABAergic interneurons $(27,28)$. Notably, postmortem studies have consistently demonstrated widespread abnormalities in cortical inhibitory GABAergic interneurons in PSZ resulting in reduced inhibition 
Barnes-Scheufler et al. Attentional Competition during VWM Encoding in Schizophrenia

(29). Additionally, neuroimaging, behavioral and computational data also provide evidence for reduced GABAergic inhibition and its central role for cognitive dysfunction (30). Therefore, impaired GABAergic inhibition in early visual areas might be a crucial mechanism underlying the insufficient suppression of attentional capture by salient stimuli in PSZ. Yet, if the use of spatial cues enables PSZ to overcome this deficit, this would indicate that enhancing inhibitory control of attentional selection exerted by frontal-parietal areas is sufficient to increase local inhibition within early visual areas. It would also suggest that impaired control of selection is the central attentional deficit.

The goal of the current study was to test these hypotheses in a behavioral VWM experiment directly contrasting top-down and bottom-up attentional processes during VWM encoding. To this end, we employed a VWM paradigm containing an equal number of salient (flickering) and non-salient (non-flickering) Gabor patches with different orientations. These were preceded by either a predictive cue indicating the location of task-relevant stimuli, or a non-predictive cue providing no such information.

\section{METHODS}

\section{Participants}

We recruited 69 PSZ from psychiatric outpatient facilities in and around Frankfurt am Main, Germany. We established diagnoses of all patients according to DSM-5 criteria based on a clinical interview and careful chart review at a consensus conference. We used the Positive and Negative Syndrome Scale (PANSS) to assess current psychopathology. All patients were on stable antipsychotic medication for at least one month at the time of the study. 
Barnes-Scheufler et al. Attentional Competition during VWM Encoding in Schizophrenia

74 healthy control subjects (HCS) were recruited by online and printed advertisements. They were screened using the German version of the Structural Clinical Interview SCID-I from the Diagnostic and Statistical Manual, Version IV (31). They reported no history of any psychiatric illness and no family history of psychiatric illness in first-degree relatives. All participants reported no history of neurological illness and no drug use (excluding nicotine) within the past six months. Groups were matched for age, sex, premorbid IQ, years of education, and parental years of education (Table 1). The ethics committee of the University Hospital Frankfurt approved all study procedures. Subjects provided written informed consent after receiving a complete description of the study.

\section{Working Memory Task}

A visuospatial change detection task (Figure 1) was implemented on a personal computer using Presentation software version 14.9 (www.neurobs.com).

Throughout the experiment a black fixation cross was presented at the center of the screen. Subsequently, either a predictive endogeneous cue or a non-predictive endogeneous cue was presented by briefly turning the fixation cross white for 300 ms. For the predictive cue, the white arms of the fixation cross indicated the future locations of the task relevant Gabor patches. For the non-predictive cue, the entire fixation cross turned white, providing no location information. After a $300 \mathrm{~ms}$ preparation interval, the encoding array was displayed for $400 \mathrm{~ms}$. It consisted of four Gabor patches shown at four fixed locations equally spaced on an imaginary circle around the fixation cross. Two of the four patches flickered at a frequency of 7.5 Hz. Before each block, participants were informed about the currently taskrelevant Gabor patches (flickering or non-flickering) and of the high likelihood that they would be probed during retrieval. In the predictive cue conditions, participants 
Barnes-Scheufler et al. Attentional Competition during VWM Encoding in Schizophrenia

therefore were provided with two sources of information about the targets: the instruction before the block and the additional cue at the start of each trial. The delay phase lasted for 2000 ms on average, with a jitter of 250 ms. During retrieval, one Gabor patch was shown within a white box at one of the four fixed locations for $3000 \mathrm{~ms}$. The other three Gabor patches were blurred out. Participants had to indicate by button press if the orientation of the highlighted Gabor patch was identical to or differed from the Gabor patch shown at the same location in the encoding array (for further details, see supplementary materials).

In $80 \%$ of trials, we probed a Gabor patch of the task-relevant type participants were instructed to focus on (target trials). In $20 \%$ of trials, we probed a Gabor patch of the task-irrelevant type participants were not instructed to focus on (catch trials). This was done to assess each participants' efficiency of attentional prioritization, which was operationalized as the difference between the amount of task-relevant and task-irrelevant information stored in WWM. We based our analysis on target trials. This resulted in four conditions: flickering / predictive cue, flickering / non-predictive cue, non-flickering / predictive cue, non-flickering / non-predictive cue. A total of 400 trials were presented, 100 for each condition, divided into four blocks of 200 trials, counterbalanced in order across participants and groups.

\section{Analysis of Behavioral Data}

The amount of information stored in WWM was quantified using Cowan's K, where K $=($ hit rate + correct rejection rate -1$) \times$ memory set size $(3,32)$. For the main analysis, we used Cowan's K for the target trials of each condition. Statistical analyses were conducted using SPSS (IBM) Version 22, and the software package R (www.r-project.org). To control for its well-established impact on VWM, we added age as a covariate. The final model included group, condition, the interaction 
Barnes-Scheufler et al. Attentional Competition during VWM Encoding in Schizophrenia

between group and condition, as well as age as a covariate (for additional details, see supplementary materials).

Significant main effects and interactions were further analyzed with pairwise t-tests, adjusted for age. P-values for the pairwise comparisons were adjusted with the Tukey method for a family of four estimates. We computed effect sizes with generalized eta squared for the ANCOVA. For the within group comparisons, Cohen's dt was calculated based on the age-adjusted t-ratio. For this the function 't_to_d' (package effectsize) was used in R. All participants had an accuracy for target trials of greater than $50 \%$.

\section{Correlation with working memory capacity}

To assess the relationship between the ability to prioritize task-relevant information and WM capacity, we correlated the effectiveness of attentional prioritization (Cowan's K for target trials minus Cowan's K for catch trials) in each participant across all four conditions with an independent estimate of VWM capacity derived from a 60-trial canonical change detection task (33). For 200 ms, Participants viewed an array of four colored circles, which were spaced equally apart on an imaginary circle around a central fixation cross (Figure S1). After an 1800 ms delay, a test array followed. Within 3000 ms, participants had to indicate by mouse-click if the test array was identical to the sample array presented during the encoding phase, or if one of the circles had changed color. Due to the use of a whole-display recognition test array, VWM capacity was calculated using Pashler's K, where K is memory set size $\times($ (hit rate - correct rejection rate -1$) /(1$ - correct rejection rate 1)) (34). 
Barnes-Scheufler et al. Attentional Competition during VWM Encoding in Schizophrenia

\section{RESULTS:}

HCS encoded more information into WWM in all four conditions (Figure 2): flickering / predictive cue $($ mean $=1.32, \mathrm{SD}=0.39)$, flickering $/$ non-predictive cue $($ mean $=$ $1.29, \mathrm{SD}=0.41)$, non-flickering / predictive cue $($ mean $=1.28, \mathrm{SD}=0.39)$, nonflickering / non-predictive cue $($ mean $=1.20, S D=0.43)$ than PSZ : flickering / predictive cue $($ mean $=1.02, S D=0.40)$, flickering $/$ non-predictive cue $($ mean $=$ $0.94, \mathrm{SD}=0.48)$, non-flickering / predictive cue (mean $=0.96, \mathrm{SD}=0.46)$, nonflickering / non-predictive cue (mean $=0.74, \mathrm{SD}=0.46)$.

\section{ANCOVA Results:}

A significant effect of group with a large effect size on Cowan's K was observed in our repeated-measures ANCOVA transformed with the non-paranormal method ( $p$ $<0.001, \eta^{2}=0.157$ ) (Table 2). As expected, a significant effect of age was also observed with a small effect size $\left(p=0.003, \eta^{2}=0.048\right)$. In addition, we observed a significant effect of condition with small effect size $\left(p<0.001, \eta^{2}=0.032\right)$, and a significant interaction of group and condition with a small effect size $\left(p=0.027, \eta^{2}=\right.$ 0.005). We did not observe a significant interaction between age and condition.

\section{Post-hoc Pairwise Comparisons:}

\section{Between Groups:}

A significant reduction of the amount of information stored in VWM was observed in PSZ compared to HCS in each condition: flickering / predictive cue $(p<0.001)$, flickering / non-predictive cue $(p<0.001)$, non-flickering / predictive cue $(p<0.001)$ and non-flickering / non-predictive cue $(p<0.001)$. 
Barnes-Scheufler et al. Attentional Competition during VWM Encoding in Schizophrenia

\section{Within Groups:}

For HCS, a significant reduction of the amount of information stored in VWM was observed in the non-flickering / non-predictive cue compared to the flickering / nonpredictive cue condition with a small effect size $(p=0.014, d t=0.3)$. Similarly, a significant reduction of stored information was observed in PSZ in the non-flickering / non-predictive cue condition as compared to the flickering / non-predictive cue condition with a moderate effect size $(p<0.001, d t=0.5)$.

Additionally, PSZ stored significantly more information in the non-flickering / predictive cue condition as compared to the non-flickering / non-predictive cue condition with a moderate effect size $(p<0.001, d t=0.5)$. Importantly, this effect was not observed in HCS. Further results can be found in Table 2.

\section{Correlation with working memory capacity}

We correlated each individual's effectiveness of attentional prioritization (Cowan's K for target trials minus Cowan's K for catch trials) across all four conditions with an independent estimate of their VWM capacity. For HCS, WM capacity did not correlate with the effectiveness of attentional prioritization across all conditions $\left(\right.$ Mean $\left.=3.31, \mathrm{SD}=0.58, r_{s}=0.16, p=0.167\right)$. For PSZ, we observed a significant correlation between WM capacity and the effectiveness of attentional prioritization across all conditions $\left(\right.$ Mean $\left.=2.91, \mathrm{SD}=0.74, r_{s}=0.25, p=0.038\right)$. 
Barnes-Scheufler et al. Attentional Competition during VWM Encoding in Schizophrenia

\section{DISCUSSION}

We studied the competition between bottom-up and top-down attentional processes during VWM encoding in PSZ and HCS in order to elucidate how disturbed attentional control contributes to VWM dysfunction in schizophrenia.

Overall, we observed a significant main effect of group and a significant interaction between group and condition after correcting for age, which also showed a significant effect. Post-hoc analyses revealed that PSZ stored significantly less information in all four task conditions compared to HCS. As expected, after presentation of a non-predictive cue PSZ stored significantly more information in the flickering compared to the non-flickering condition. Notably, HCS showed the same effect, albeit with a small effect size compared to a moderate effect size for PSZ. Importantly, only PSZ but not HCS were able to store significantly more information in the non-flickering condition when shown a predictive cue compared to a nonpredictive cue. Finally, after presentation of a predictive cue there was no significant difference for the amount of stored information for the flickering and non-flickering condition in either HCS or PSZ.

We could replicate a previous report by Hahn and colleagues of a bottom-up attentional bias toward salient distractors during VWM encoding in PSZ (5). The central question of our study was concerned with the effect of a predictive cue on the processing of salient distractors in PSZ. For the non-flickering conditions, where patients had to suppress highly salient stimuli, the addition of a predictive cue lead to a significant increase in the amount of stored information only in PSZ. This indicates that patients were able to utilize external cues to enhance prioritization of task-relevant information. Within the context of predictive coding, it points to an improved precision of high-level priors and an intact ability to use those priors in PZS in order to implement attentional selection and correctly prioritize stimuli for 
Barnes-Scheufler et al. Attentional Competition during VWM Encoding in Schizophrenia

encoding into VWM. It remains an open question whether the use of feature-based instead of spatial-based cues can lead to a similar beneficial effect. Our findings also support the notion that insufficiently precise and stable priors are a primary cause of impaired attentional control during VWM encoding in schizophrenia. Additionally, we observed a significant correlation between WM capacity and the effectiveness of attentional prioritization across all four conditions in PSZ but not in HCS. This highlights the importance of impaired attentional prioritization for VWM dysfunction in schizophrenia.

Furthermore, the presentation of a predictive cue enhanced the suppression of irrelevant salient information in PSZ, which indicates a strengthening of inhibitory mechanisms. This suggests that disturbed inhibition in PSZ thought to underlie impaired control of attentional selection is not an irreversible phenomenon, but that it can be partially counteracted by aiding the attentional set with spatial cues.

Additionally, our results are compatible with the interpretation that enhanced inhibitory control of attentional selection might also increase local inhibition within early visual areas.

Unexpectedly, we also observed a weak bottom-up attentional bias toward salient distractors in HCS. This finding points to the existence of a bottom-up attentional bias in healthy subjects when challenged by highly salient distractors. Hahn and colleagues did not observe this subtle effect, a discrepancy that might be attributable to differences in task design. While both paradigms probed WWM, participants in the previous study had to memorize the position of flickering or nonflickering checkerboard stimuli. Conversely, participants in our study had to memorize the fine-grained orientation of Gabor patches. Manipulation of stimulus salience by flickering might have interfered to a greater degree with perceptual processing of Gabor patch orientation than with checkerboard orientation. 
Barnes-Scheufler et al. Attentional Competition during VWM Encoding in Schizophrenia

Furthermore, Hahn and colleagues employed a whole-display recognition paradigm, while we used a partial-display recognition paradigm, which typically is cognitively more demanding.

Our results imply that a bottom-up attentional bias toward salient information is not a unique feature of schizophrenia. Yet, we also observed a significant reduction in the amount of stored information for the non-flickering / non-predictive cue condition in PSZ compared to HCS. This indicates that PSZ are considerably more susceptible to failures of implementing selection in the presence of salient distractors without the aid of an external cue than HCS. This results in greater interference with the prioritization of information during VWM encoding in PSZ. Notably, another study investigating distractor processing during VWM encoding in PSZ did not report evidence for a bottom-up attentional bias (35). Here, stationary rotating motion was used to generate salient distractor stimuli. However, this manipulation of stimulus properties might not have been sufficiently salient to trigger automatic attentional capture, especially in light of the extensive evidence for motion processing impairments in PSZ (36). By contrast, flickering of visual stimuli has been shown to be particularly salient (37).

Importantly, there is converging evidence of attentional hyperfocusing during visual information processing in PSZ (38). Increased vulnerability to attentional capture by salient distractors in PSZ could also be interpreted as a manifestation of attentional hyperfocusing, i.e. hyperfocusing on irrelevant information. This could imply a shared mechanism involving abnormal GABA-mediated inhibition for both phenomena. However, the exact relationship between the failure to implement attentional selection in the presence of salient visual distractors and the degree of attentional hyperfocusing in PSZ remains to be investigated. As attentional hyperfocusing appears to constrain the amount of information PSZ can encode into 
Barnes-Scheufler et al. Attentional Competition during VWM Encoding in Schizophrenia

VWM (39), it is conceivable that it contributed to the general VWM impairment we observed in PSZ across all task conditions. However, we were not able to make any inferences regarding this interpretation based on our current paradigm.

To summarize, the current paradigm allowed us to study two central attentional processes, the control of attention and the implementation of selection and their contribution to VWM encoding deficits in PSZ. We were able to delineate the extent of intact and impaired aspects of these processes and to provide novel constraints for models of attentional dysfunction in the context of VWM. The neurophysiological underpinnings of our findings should be illuminated using functional neuroimaging. The relevance of such a study is underscored by the inclusion of both attention and working memory as constructs in the cognition domain of the Research Domain Criteria initiative (40). Given the presence of deficits in attention and working memory in other neuropsychiatric disorders such as bipolar disorder, our paradigm might therefore be useful for studying the pathophysiology of impaired information across diagnostic categories.

\section{Acknowledgements}

The authors are very thankful to Prof. Dr. Christoph Fehr, Dr. Peter Hustedt for their support in recruiting patients, , as well as to Deliah Macht, Lisa Goldschmidt, Tobias Lehmann and Caroline Passow for assisting with data acquisition. 
bioRxiv preprint doi: https://doi.org/10.1101/2021.05.19.442954; this version posted May 19, 2021. The copyright holder for this preprint (which was not certified by peer review) is the author/funder. All rights reserved. No reuse allowed without permission.

\section{Barnes-Scheufler et al. Attentional Competition during VWM Encoding in Schizophrenia}

\section{References}

1. Mayer JS, Bittner RA, Nikolic D, Bledowski C, Goebel R, Linden DE. Common neural substrates for visual working memory and attention. NeuroImage. 2007 Jun;36(2):441-53. PubMed PMID: 17462914.

2. Gazzaley A, Nobre AC. Top-down modulation: bridging selective attention and working memory. Trends in cognitive sciences. 2012 Feb;16(2):129-35. PubMed PMID: 22209601. Pubmed Central PMCID: 3510782.

3. Cowan N. The magical number 4 in short-term memory: A reconsideration of mental storage capacity. Behavioral and brain sciences. 2001;24(1):87-114.

4. Carter CS, Barch DM. Cognitive neuroscience-based approaches to measuring and improving treatment effects on cognition in schizophrenia: the CNTRICS initiative. Schizophrenia bulletin. 2007 Sep;33(5):1131-7. PubMed PMID: 17630405. Pubmed Central PMCID: 2632368.

5. Hahn B, Robinson BM, Kaiser ST, Harvey AN, Beck VM, Leonard CJ, et al. Failure of schizophrenia patients to overcome salient distractors during working memory encoding. Biological psychiatry. 2010 Oct 1;68(7):603-9. PubMed PMID: 20570242. Pubmed Central PMCID: 2942999.

6. Mayer JS, Park S. Working memory encoding and false memory in schizophrenia and bipolar disorder in a spatial delayed response task. J Abnorm Psychol. 2012;121(3):784.

7. Hartman M, Steketee MC, Silva S, Lanning K, McCann H. Working memory and schizophrenia: evidence for slowed encoding. Schizophr Res. 2003;59(2-3):99-113.

8. Haenschel C, Bittner RA, Haertling F, Rotarska-Jagiela A, Maurer K, Singer W, et al. Contribution of impaired early-stage visual processing to working memory dysfunction in adolescents with schizophrenia: a study with event-related potentials and functional magnetic resonance imaging. Arch Gen Psychiatry. 2007 Nov;64(11):1229-40. PubMed PMID: 17984392. eng.

9. Bittner RA, Linden DE, Roebroeck A, Hartling F, Rotarska-Jagiela A, Maurer K, et al. The When and Where of Working Memory Dysfunction in Early-Onset Schizophrenia-A Functional Magnetic Resonance Imaging Study. Cerebral cortex. 2015 Sep;25(9):2494-506. PubMed PMID: 24675869.

10. Vogel EK, McCollough AW, Machizawa MG. Neural measures reveal individual differences in controlling access to working memory. Nature. 2005;438(7067):500.

11. Cowan N, Morey CC. Visual working memory depends on attentional filtering. Trends in cognitive sciences. 2006;10(4):139-41.

12. Lorenc ES, Mallett R, Lewis-Peacock JA. Distraction in Visual Working Memory: Resistance is Not Futile. Trends in cognitive sciences. 2021.

13. Liesefeld HR, Liesefeld AM, Sauseng P, Jacob SN, Müller HJ. How visual working memory handles distraction: cognitive mechanisms and electrophysiological correlates. Visual Cognition. 2020;28(5-8):372-87. 14. Desimone R, Duncan J. Neural mechanisms of selective visual attention. Annual review of neuroscience. 1995;18:193-222. PubMed PMID: 7605061.

15. Luck SJ, Gold JM. The construct of attention in schizophrenia. Biological psychiatry. 2008 Jul 1;64(1):34-9. PubMed PMID: 18374901. Pubmed Central PMCID: 2562029.

16. Fecteau JH, Munoz DP. Salience, relevance, and firing: a priority map for target selection. Trends in cognitive sciences. 2006;10(8):382-90.

17. Corbetta M, Shulman GL. Control of goal-directed and stimulus-driven attention in the brain. Nature reviews neuroscience. 2002;3(3):201-15.

18. Gaspelin N, Luck SJ. Combined Electrophysiological and Behavioral Evidence for the Suppression of Salient Distractors. Journal of cognitive neuroscience. 2018 Sep;30(9):1265-80. PubMed PMID: 29762104.

19. Sawaki R, Luck SJ. Capture versus suppression of attention by salient singletons:

Electrophysiological evidence for an automatic attend-to-me signal. Attention, Perception, \& Psychophysics. 2010;72(6):1455-70.

20. Friston K, Kiebel S. Predictive coding under the free-energy principle. Philosophical Transactions of the Royal Society B: Biological Sciences. 2009;364(1521):1211-21.

21. Sterzer P, Adams RA, Fletcher P, Frith C, Lawrie SM, Muckli L, et al. The predictive coding account of psychosis. Biological psychiatry. 2018;84(9):634-43.

22. Fuller RL, Luck SJ, Braun EL, Robinson BM, McMahon RP, Gold JM. Impaired control of visual attention in schizophrenia. Journal of abnormal psychology. 2006 May;115(2):266-75. PubMed PMID: 16737391.

23. Mayer JS, Fukuda K, Vogel EK, Park S. Impaired contingent attentional capture predicts reduced working memory capacity in schizophrenia. PloS one. 2012;7(11):e48586.

24. Gold JM, Fuller RL, Robinson BM, McMahon RP, Braun EL, Luck SJ. Intact attentional control of working memory encoding in schizophrenia. Journal of abnormal psychology. 2006;115(4):658.

25. Gold JM, Hahn B, Strauss GP, Waltz JA. Turning it upside down: areas of preserved cognitive function in schizophrenia. Neuropsychology review. 2009 Sep;19(3):294-311. PubMed PMID: 19452280. Pubmed Central PMCID: 3746345. 
bioRxiv preprint doi: https://doi.org/10.1101/2021.05.19.442954; this version posted May 19, 2021. The copyright holder for this preprint (which was not certified by peer review) is the author/funder. All rights reserved. No reuse allowed without permission.

\section{Barnes-Scheufler et al. Attentional Competition during VWM Encoding in Schizophrenia}

26. Gaspelin N, Luck SJ. The role of inhibition in avoiding distraction by salient stimuli. Trends in cognitive sciences. 2018;22(1):79-92.

27. Zhang S, Xu M, Kamigaki T, Do JPH, Chang W-C, Jenvay S, et al. Long-range and local circuits for top-down modulation of visual cortex processing. Science. 2014;345(6197):660-5.

28. Buschman TJ, Kastner S. From behavior to neural dynamics: an integrated theory of attention. Neuron. 2015;88(1):127-44.

29. Lewis DA. Inhibitory neurons in human cortical circuits: substrate for cognitive dysfunction in schizophrenia. Curr Opin Neurobiol. 2014;26:22-6.

30. Shaw AD, Knight L, Freeman TC, Williams GM, Moran RJ, Friston KJ, et al. Oscillatory, computational, and behavioral evidence for impaired GABAergic inhibition in schizophrenia. Schizophr Bull. 2020;46(2):345-53.

31. Saß H, Wittchen H-U, Zaudig M, Houben I. Dsm-iv-tr. Diagnostische Kriterien Hogrefe, Göttingen. 2003.

32. Rouder JN, Morey RD, Morey CC, Cowan N. How to measure working memory capacity in the change detection paradigm. Psychonomic Bulletin \& Review. 2011;18(2):324-30.

33. Barnes-Scheufler CV, Passow C, Rösler L, Mayer JS, Oertel V, Kittel-Schneider S, et al. Transdiagnostic comparison of visual working memory capacity in bipolar disorder and schizophrenia. International Journal of Bipolar Disorders. 2021;9(1):1-12.

34. Pashler H. Familiarity and visual change detection. Perception \& psychophysics. 1988;44(4):369-78. 35. Erickson MA, Hahn B, Leonard CJ, Robinson B, Gray B, Luck SJ, et al. Impaired working memory capacity is not caused by failures of selective attention in schizophrenia. Schizophrenia bulletin. 2015 Mar;41(2):366-73. PubMed PMID: 25031223. Pubmed Central PMCID: 4332943.

36. Chen Y. Abnormal visual motion processing in schizophrenia: a review of research progress. Schizophrenia bulletin. 2011;37(4):709-15.

37. Merigan WH, Maunsell JH. How parallel are the primate visual pathways? Annual review of neuroscience. 1993;16(1):369-402.

38. Luck SJ, Leonard CJ, Hahn B, Gold JM. Is Attentional Filtering Impaired in Schizophrenia? Schizophr Bull. 2019;45(5):1001-11.

39. Luck SJ, Hahn B, Leonard CJ, Gold JM. The hyperfocusing hypothesis: A new account of cognitive dysfunction in Schizophrenia. Schizophr Bull. 2019;45(5):991-1000.

40. Insel T, Cuthbert B, Garvey M, Heinssen R, Pine DS, Quinn K, et al. Research domain criteria (RDoC): toward a new classification framework for research on mental disorders. Am Psychiatric Assoc; 2010. 
Barnes-Scheufler et al. Attentional Competition during VWM Encoding in Schizophrenia

\section{Figure Legends:}

Figure 1. Visual change detection task with four conditions; flickering / predictive cue, flickering / non-predictive cue, non-flickering / predictive cue, non-flickering / non-predictive cue. Flickering is indicated by white dashes around stimuli. The set size of four items was kept constant.

Figure 2. Amount of information stored in VWM - estimated with Cowan's K - in PSZ and HCS displaying within and between group contrasts. * Indicates $p<0.05$, ${ }^{* * *}$ indicates $p<0.001$ Error bars indicate standard deviation. Raw data is pictured, statistical results reflect transformed data. For visualization of transformed data, please see supplementary materials.

\section{Table Legends:}

Table 1. Values are mean (SD), or $n$. All statistics reported are 2-tailed. FGA = firstgeneration antipsychotics, SGA = second-generation antipsychotics .

Table 2. Degrees of freedom are corrected with Greenhouse-Geisser. 
Retrieval

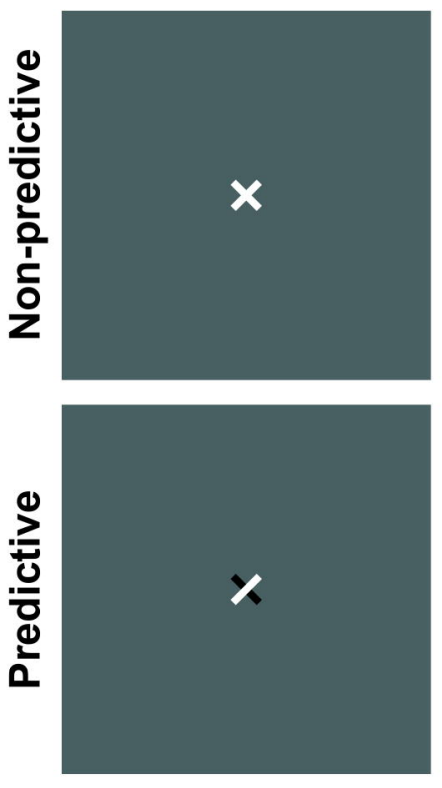

Non-flickering targets

$300 \mathrm{~ms}$

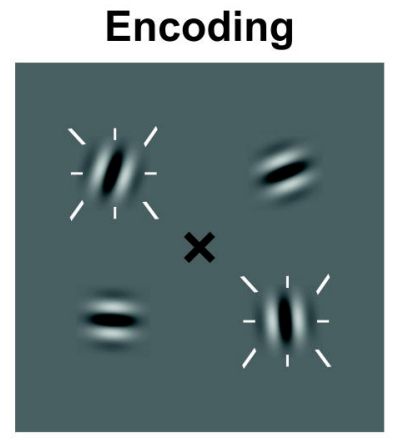

$400 \mathrm{~ms}$

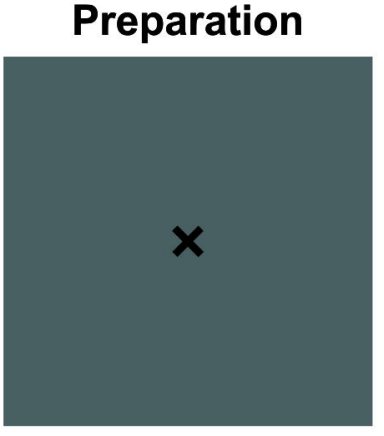

$300 \mathrm{~ms}$
Delay

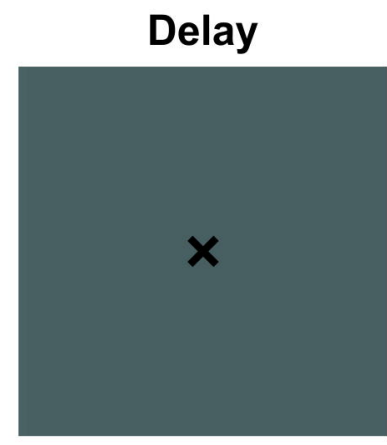

$2000 \mathrm{~ms}$

\section{Flickering targets}

$x$

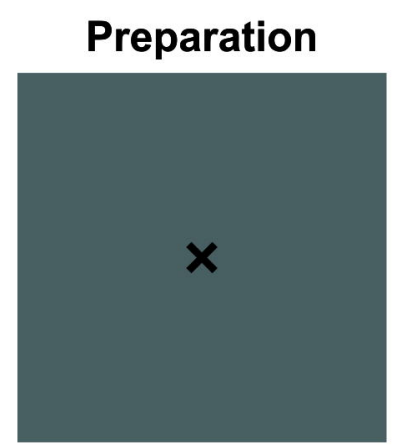

$300 \mathrm{~ms}$

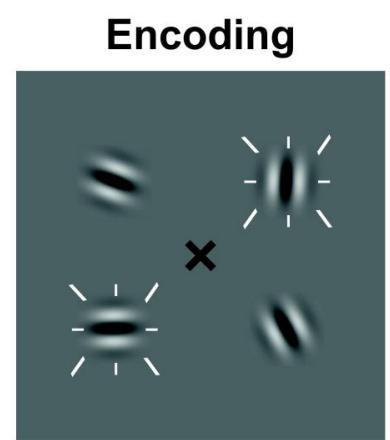

$400 \mathrm{~ms}$
Retrieval

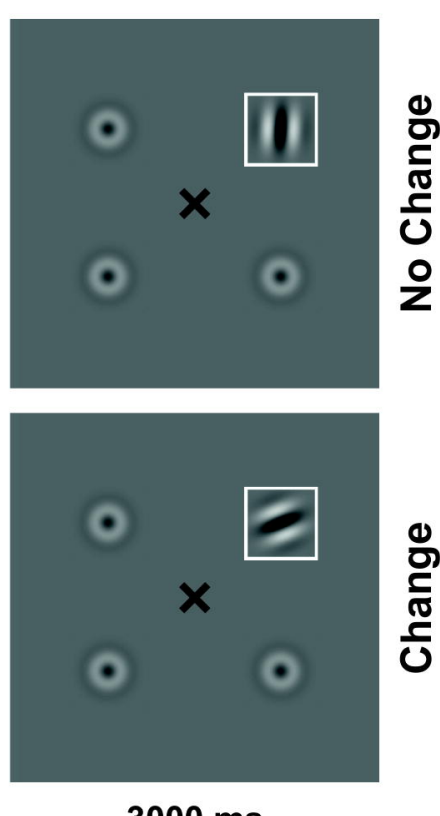

$3000 \mathrm{~ms}$
$2000 \mathrm{~ms}$

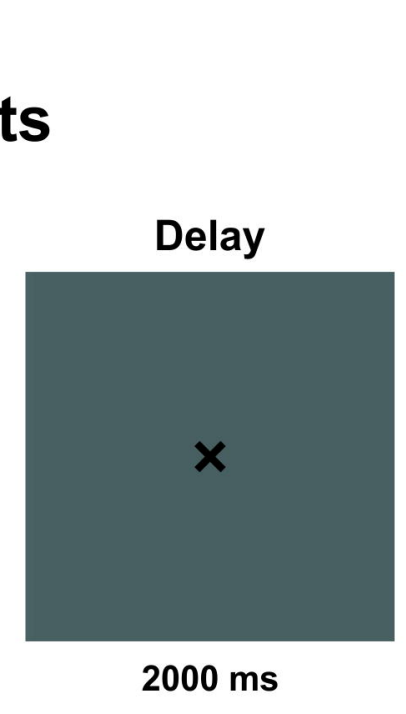

$\frac{0}{\frac{0}{0}}$

$3000 \mathrm{~ms}$

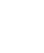

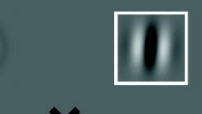


Table 1. Participant Characteristics

\begin{tabular}{|c|c|c|c|c|}
\hline & HCS $(n=74)$ & PSZ $(n=69)$ & Statistic & p Value \\
\hline \multicolumn{5}{|l|}{ Demographics } \\
\hline Sex, Male/Female & $45 / 29$ & $45 / 24$ & Fisher's Exact Test & $p=0.607$ \\
\hline Parental Education, Years & $14.93(3.02)$ & $14.65(3.37)$ & $\mathrm{t}_{141}=-0.53$ & $p=0.600$ \\
\hline Premorbid IQ & $113.93(11.16)$ & $110.00(13.25)$ & $\mathrm{t}_{141}=-1.92$ & $p=0.056$ \\
\hline Schizophrenia & & 45 & & \\
\hline Schizoaffective disorder & & 24 & & \\
\hline \multicolumn{5}{|l|}{ Psychopathology } \\
\hline PANSS Positive & & $10.09(3.50)$ & & \\
\hline PANSS Negative & & $11.80(3.85)$ & & \\
\hline PANSS General & & $22.82(4.86)$ & & \\
\hline PANSS Total & & $44.56(9.84)$ & & \\
\hline \multicolumn{5}{|l|}{ Medication } \\
\hline Valproate & & 2 & & \\
\hline Antidepressants & & 16 & & \\
\hline
\end{tabular}


Table 2. Results

\begin{tabular}{llll}
\hline Analysis & Statistic & $p$ Value & Effect Slze \\
\hline ANCOVA & & & \\
Group & $\mathrm{F}_{1,140}=32.85$ & $p<0.001$ & $\eta^{2}=0.157$ \\
Age & $\mathrm{F}_{1,140}=8.90$ & $p=0.003$ & $\eta^{2}=0.048$ \\
Condition & $\mathrm{F}_{3,411}=22.81$ & $p<0.001$ & $\eta^{2}=0.032$ \\
Group x Condition & $\mathrm{F}_{3,411}=3.10$ & $p=0.027$ & $\eta^{2}=0.005$ \\
Age x Condition & $\mathrm{F}_{3,411}=0.44$ & $p=0.717$ & $\eta^{2}<.001$ \\
& & & \\
Post-hoc Between Group Comparisons & & & \\
Flickering / predictive cue & $\mathrm{t}_{140}=4.53$ & $p<0.001$ & \\
Flickering / non-predictive cue & $\mathrm{t}_{140}=4.90$ & $p<0.001$ & \\
Non-flickering / predictive cue & $\mathrm{t}_{140}=4.75$ & $p<0.001$ & \\
Non-flickering / non-predictive cue & $\mathrm{t}_{140}=6.36$ & $p<0.001$ & \\
& & & \\
Post-hoc Within Group Comparisons & & & \\
HCS & & & \\
Flickering / predictive cue vs Flickering / non-predictive cue & $\mathrm{t}_{140}=0.55$ & $p=0.948$ & $d t=0.1$ \\
Flickering / predictive cue vs Non-flickering / predictive cue & $\mathrm{t}_{140}=1.31$ & $p=0.558$ & $d t=0.1$ \\
Non-flickering / predictive cue vs Non-flickering / non-predictive cue & $\mathrm{t}_{140}=2.24$ & $p=0.117$ & $d t=0.2$ \\
Non-flickering / non-predictive cue vs. Flickering / non-predictive cue & $\mathrm{t}_{140}=3.06$ & $p=0.014$ & $d t=0.3$ \\
& & & \\
PSZ & & & \\
Flickering / predictive cue vs Flickering / non-predictive cue & $\mathrm{t}_{140}=1.60$ & $p=0.390$ & $d t=0.1$ \\
Flickering / predictive cue vs Non-flickering / predictive cue & $\mathrm{t}_{140}=1.52$ & $p=0.431$ & $d t=0.1$ \\
Non-flickering / predictive cue vs Non-flickering / non-predictive cue & $\mathrm{t}_{140}=5.70$ & $p<0.001$ & $d t=0.5$ \\
Non-flickering / non-predictive cue vs. Flickering / non-predictive cue & $\mathrm{t}_{140}=5.66$ & $p<0.001$ & $d t=0.5$ \\
& & & \\
\hline
\end{tabular}

